\title{
Effect of cooling water on ablation in Er:YAG laserosteotome of hard bone
}

Hamed Abbasi, Lina Beltrán, Georg Rauter, Raphael Guzman, Philippe C. Cattin, et al.

Hamed Abbasi, Lina Beltrán, Georg Rauter, Raphael Guzman, Philippe C. Cattin, Azhar Zam, "Effect of cooling water on ablation in Er:YAG laserosteotome of hard bone," Proc. SPIE 10453, Third International Conference on Applications of Optics and Photonics, 104531I (22 August 2017); doi: 10.1117/12.2272138

SPIE Event: Third International Conference on Applications of Optics and Photonics, 2017, Faro, Portugal 


\title{
Effect of Cooling Water on Ablation in Er:YAG Laserosteotome of Hard Bone
}

\author{
Hamed Abbasi*a, Lina Beltrán ${ }^{\mathrm{a}}$, Georg Rauter ${ }^{\mathrm{b}}$, Raphael Guzman ${ }^{\mathrm{c}}$, Philippe C. Cattin ${ }^{\mathrm{d}}$, Azhar Zam*a \\ ${ }^{a}$ Biomedical Laser and Optics Group, Department of Biomedical Engineering, University of Basel, \\ Gewerbestrasse 14, CH-4123 Allschwil, Switzerland \\ ${ }^{\mathrm{b}}$ Bio-Inspired RObots for Medicine-Lab., Department of Biomedical Engineering, University of \\ Basel, Gewerbestrasse 14, CH-4123 Allschwil, Switzerland \\ ${ }^{\mathrm{c}}$ Department of Neurosurgery, University Hospital Basel, Basel, Switzerland \\ ${ }^{\mathrm{d}}$ Center for Medical Image Analysis and Navigation, Department of Biomedical Engineering, \\ University of Basel, Gewerbestrasse 14, CH-4123 Allschwil, Switzerland
}

\begin{abstract}
The aim of this paper is to examine the effect of pig bone immersion in different levels of cooling water during laser ablation with a Er:YAG laser. The laser worked at $2940 \mathrm{~nm}$ wavelength and $10 \mathrm{~Hz}$ repetition rate in microseconds pulse duration regime. The bone was immersed in different levels of cooling water in a sample container for preventing carbonization. The bone samples were ablated with fixed deposited energy to investigate at which water level Er:YAG lasers start ablating bone through a layer of water. Results showed that the maximum level of water that laser can pass through to start the ablation nonlinearly depends on pulse energy.
\end{abstract}

Keywords: Laserosteotome, ablation, carbonization, cooling water, porcine bone, Er:YAG, microsecond pulse, minimally invasive surgery

\section{INTRODUCTION}

Smart surgical tools are devices that can help surgeons reduce possible medical errors. Reducing medical errors are important [1]. Some of these lethal medical errors can occur during surgery; therefore, developing smart surgical tools that prevent medical errors is necessary. A laserosteotome would be one such smart surgical system that can help surgeons in cutting hard and soft tissues in a safe and efficient manner. Laserosteotomes are applied in the fields of orthopedics, craniomaxillofacial surgery, neurosurgery, otolaryngology, traumatology, and spine surgery. By performing this kind of minimal invasive osteotomies (bone cuts) the duration of hospital stays and rehabilitation is expected to be reduced. In comparison with mechanical tools, it has been proven that laserosteotomes have significant advantages such as non-contact interaction, minimal invasiveness, precise and small cuts based on pre-operative planning, functional cut geometry (smart cuts), accelerated healing, and less trauma [2-4]. However, without using any cooling agent soon after starting the ablation the bone would start to carbonize and bone healing will be impeded, therefore some kind of cooling system is necessary in combination with laserosteotome. In our current setup, we use an Er:YAG laser because it is one of the best lasers for cutting the bones [2]. The principle of bone ablation with Er:YAG lasers is based on laser energy absorption by water in the bone. So simply immersing the bone in water for cooling will only lead to an evaporation of the surrounding water, but not to cutting of bone. This behavior strongly depends on the wavelength of the laser. Lasers at other wavelengths would allow bone cutting inside water [5]. Moreover, it has been shown that different water cooling conditions of spray systems in Er:YAG laser ablation of hard dental tissues influence ablation efficiency [6]. The aim of this paper is to investigate limitation of water cooling conditions (maximum level of cooling water) that would still allow laser ablation with Er:YAG lasers. Knowing the maximum water level that still allows bone ablation is important for adjusting the diameter of the cooling water droplet in spray systems or the height of water in a running water systems for irrigation in Er:YAG laserosteotomes.

\footnotetext{
*hamed.abbasi@unibas.ch; phone +41 (0)61 20754 61; http://dbe.unibas.ch/blog

*azhar.zam@unibas.ch; phone +41 (0)61 20754 60; http://dbe.unibas.ch/blog
}

Third International Conference on Applications of Optics and Photonics, edited by Manuel F. M. Costa, Proc. of SPIE Vol. 10453, 104531I · @ 2017 SPIE · CCC code: 0277-786X/17/\$18 · doi: 10.1117/12.2272138 


\section{METHODOLOGY}

\subsection{Bone sample}

In this paper, fresh porcine bone parts from the femur of Swiss pigs were used to minimize the post-mortem changes as much as possible. The bones were kept in the deep freezer between the scarification of the animal to the starting day of the experiment. The temperature of the freezer was set to $-18^{\circ}$ centigrade. Four hours before the starting of the experiment, the bones were moved from freezer to the refrigerator which was set to $+4^{\circ}$ centigrade. Soft tissues were removed from the surface of the bones using a surgical scalpel.

\subsection{Laser}

A pulsed Er:YAG (Erbium-doped yttrium aluminium garnet) laser which emits an infrared beam at $2940 \mathrm{~nm}$ was used in this experiment. The laser is a flash lamp pumped Er:YAG laser that emits pulses in the microseconds regime. The laser could emit a laser beam with a repetition rate in the range of 10 to $50 \mathrm{~Hz}$. In this paper, the repetition rate was set to 10 Hz. The output pulse energy of the laser was adjustable. Moreover, the pulse duration of the laser was adjustable, but not in an independent manner, as soon as applying any change to the pulse duration, the pulse energy of the laser was changed and vice versa. We selected seven different pulse energies (7 different pulse durations) for the experiment in which the specification can be seen in Table 1 .

Table 1. Parameters of the experiments.

\begin{tabular}{|c|c|c|c|c|c|}
\hline \multirow{2}{*}{$\begin{array}{c}\text { Number of } \\
\text { the } \\
\text { experiment }\end{array}$} & $\begin{array}{c}\text { Pulse energy } \\
{[\mathrm{mJ}]}\end{array}$ & $\begin{array}{c}\text { Pulse duration } \\
{[\mu \mathrm{s}]}\end{array}$ & $\begin{array}{c}\text { Pulse Power } \\
{[\mathrm{kW}]}\end{array}$ & $\begin{array}{c}\text { Repetition rate } \\
{[\mathrm{Hz}]}\end{array}$ & $\begin{array}{c}\text { Average power } \\
{[\mathrm{W}]}\end{array}$ \\
\hline 1 & 100 & 201 & 0.50 & 10 & 1.0 \\
\hline 2 & 160 & 246 & 0.65 & 10 & 1.6 \\
\hline 3 & 220 & 286 & 0.77 & 10 & 2.2 \\
\hline 4 & 260 & 321 & 0.81 & 10 & 2.6 \\
\hline 5 & 300 & 360 & 0.83 & 10 & 3.0 \\
\hline 6 & 340 & 402 & 0.85 & 10 & 3.4 \\
\hline 7 & 380 & 436 & 0.87 & 10 & 3.8 \\
\hline
\end{tabular}

The pulse energy of the laser was measured using a power meter (ES220C sensor connected to PM100D meter, Thorlabs). Moreover, the pulse duration and repetition rate of the laser were checked using a PbSe fixed gain detector (PDA20H, Thorlabs) connected to a precise scopemeter (Fluke 190-504). An IR reflective neutral density filter (NDIR20A, Thorlabs) and an IR band pass filter (FB3000-500, Thorlabs) were used for reducing the power at the detector and filtering the flash pump pollution, respectively, during measurement of the pulse durations and repetition rates.

\subsection{Optical setup}

The laser line was focused on the surface of the bones using an uncoated CaF2 plano-convex lens (WG51050, Thorlabs) with a focal length of $+80.0 \mathrm{~mm}$. This focusing system provides a spot size in the order of a couple of hundreds of micrometers at the focal length of the lens. A broadband sapphire window with an anti-reflection coating (WG31050-D, Thorlabs) was put right after the lens to prevent any possible damage to the surface of the lens. The laser line was vertically directed to the surface of the bone from above by placing a gold mirror (ME05-M01, Thorlabs) between the laser head and the focusing lens, while bones were in a cylindrical container with a diameter of $77 \mathrm{~mm}$. Figure 1. Shows the schematic of the experimental setup. 


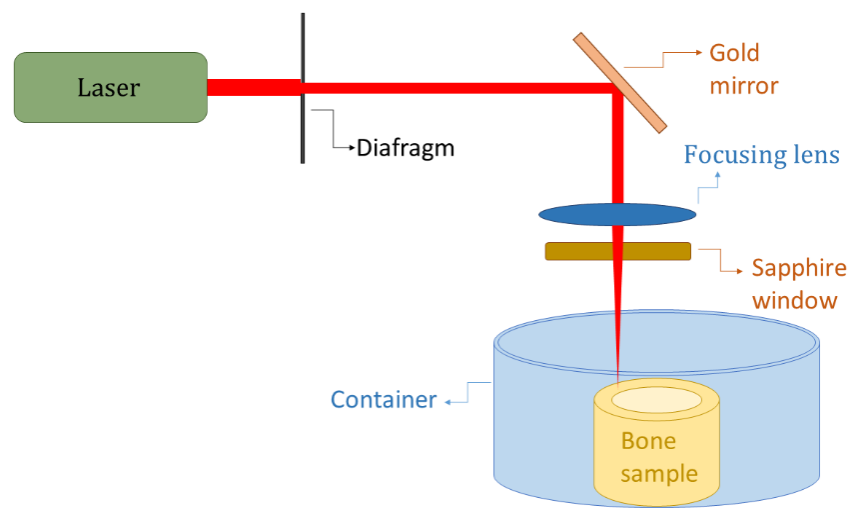

Figure 1. The schematic of the experimantal setup.

A precise syringe was used to add/remove the water into the container for realizing different heights of water above the bone. The laser pulses were started as soon as the bone was covered with $2 \mathrm{~mm}$ of water above the surface. Over time, the water was gradually removed from the container. As soon as an ablation process was initiated (observed) the extraction of water was stopped and the height of the water over the bone was calculated based on the amount of extracted water. In this way, the maximum height of water through which the laser can initiate bone ablation was obtained.

\section{RESULTS}

It is clear that the more energy is applied the higher is the ablation efficiency. For example, we measured the ablated volume of the bones with three different energies, 220, 260 and $310 \mathrm{~mJ}$ after 5 seconds of laser radiation and ablated volumes were $0.25,0.38$ and $0.61 \mathrm{~mm}^{3}$ respectively. For these three different energies, $114 \mathrm{~mL} / \mathrm{min}$ water spray was used as cooling system. The ablated volumes were measured using a microCT (computed tomography, Skyscan) system. The resolution of the CT system was isotropically $18 \mu \mathrm{m}$. Mainly, this paper is going to answer the question that what is the maximum height of water that laser can pass through to start the ablation process. Figure 2 shows the diagram of different applied energies (in the horizontal axis) vs. height of the water above the bone (in the vertical axis). Each experiment was done three times and each repetition was done on a different bone. The error bar is also depicted in the diagram. As becomes clear in the diagram, the maximum height of the water increases nonlinearly by increasing the energy. A polynomial fit which is visible in the diagram with red color shows nonlinearity of the behavior.

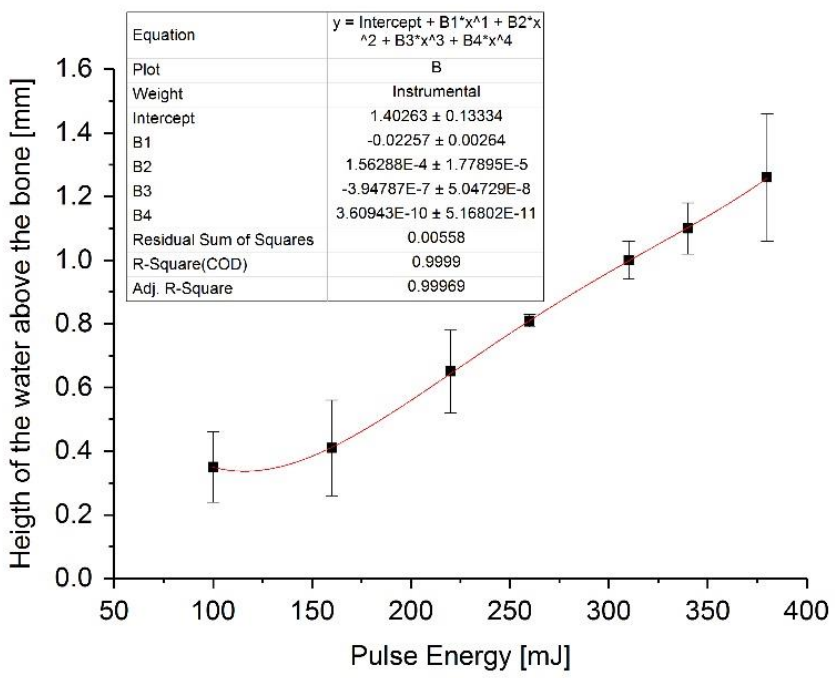

Figure 2. The height of the water above the bone for different applied pulse energies. 


\section{CONCLUSION}

Laser pulses with higher energy leads to a higher ablation efficiency (ablated bone volume per laser pulse). In this experiment, more volume was ablated at higher energy levels, as expected. However, the aim of this paper was not to measure the ablation efficiency. The main aim of this paper was to find the maximum height of water that laser can pass through to start the ablation process. It was observed that the maximum height of the water above the bone increased by increasing the energy in a nonlinear manner. The obtained data is useful to design any kind of irrigation cooling system. The diameter of the droplet of water in spray system or height of water in running water mode can be determined using these results. We observed that without using cooling water, the bone immediately starts to carbonize and therefore some kind of cooling system is necessary for any laserosteotome. It could be observed that the maximum height of the water above the bone to start the ablation process is in the order of $1 \mathrm{~mm}$. This low height of water is not enough to cover/fill the spot again with water after the first couple of laser pulses. The reasons seem to be that the generated air pressure close to the spot point of the ablation expels water; also, the bone surface is hydrophobe and prevents water from entering the ablation spot. Therefore, the irrigation system should be either realized in form of a running water or a spray system. The obtained laser-water interaction result of this paper can help to design any different cooling systems for Er:YAG laserosteotomes.

\section{ACKNOWLEDGMENTS}

The authors gratefully acknowledge funding of the Werner Siemens Foundation through the MIRACLE project. Moreover, the authors gratefully acknowledge Mr. Marek Zelechowski for his help in code writing to operate the laser.

\section{REFERENCES}

[1] Makary, M.A. and Daniel, M., "Medical error - the third leading cause of death in the US, " Bmj, 353, 2139-2139 (2016).

[2] Jowett, N., Wöllmer, W., Reimer, R., Zustin, J., Schumacher, U., Wiseman, P.W., Mlynarek, A.M., Böttcher, A., Dalchow, C.V., Lörincz, B.B. and Knecht, R., "Bone ablation without thermal or acoustic mechanical injury via a novel picosecond infrared laser (PIRL), "Otolaryngology--Head and Neck Surgery, 150(3), 385-393 (2014).

[3] Baek, K.W., Deibel, W., Marinov, D., Griessen, M., Dard, M., Bruno, A., Zeilhofer, H.F., Cattin, P. and Juergens, P., "A comparative investigation of bone surface after cutting with mechanical tools and Er:YAG laser," Lasers in surgery and medicine, 47(5), 426-432 (2015).

[4] Beltrán, L., Abbasi, H., Rauter, G., Friederich, N. F., Cattin, P. and Zam. A. "Effect of laser pulse duration on ablation efficiency of hard bone in microseconds regime" the III International Conference on Applications in Optics and Photonics (2017).

[5] Tulea, C.A., Caron, J., Gehlich, N., Lenenbach, A., Noll, R. and Loosen, P., "Laser cutting of bone tissue under bulk water with a pulsed ps-laser at $532 \mathrm{~nm}$, "Journal of biomedical optics, 20(10), 105007-105007 (2015).

[6] Kuščer, L. and Diaci, J., "Measurements of erbium laser-ablation efficiency in hard dental tissues under different water cooling conditions, " Journal of biomedical optics, 18(10), 108002-108002 (2013). 